\title{
Research on Design and Implementation of ZigBee Gateway System Based on Internet of Things
}

\author{
ZHOU Huachun ${ }^{1, \text { a }}$ \\ ${ }^{1}$ Department of the internet of things ,Chongqing College of electronic engineering, ChongQing, \\ 401331, China \\ a420749294@qq.com
}

\begin{abstract}
Keywords: Gateway; ZigBee; Internet of Things; TCP / IP
\end{abstract}
\begin{abstract}
The development of networking technology have a significant impact on society and the economy of the whole country, the ZigBee network connects with the Internet, can be controlled to a ZigBee network device in every corner of the world, it is conducive to the further development of ZigBee technology. ZigBee gateway via the Internet and the media do, it makes the monitor different regions worldwide can share information, which greatly reduces the time and space away from the physical world. In this paper, the gateway to the needs of Internet of Things are analyzed, and based on CC2530 development kit, designed and implemented a prototype system Internet of Things ZigBee gateway, complete ZigBee network management, protocol conversion, data transmission and other functions, the various communication modules and connectivity gateways whole were tested, the results showed that the ZigBee gateway enables communication between network nodes and the Internet, has better practicability.
\end{abstract}

\section{Theoretical Introduction of ZigBee}

ZigBee protocol is a short-range, low-power, low data rate wireless communication protocol, physical layer is compatible with IEEE 802. 15. 4 protocol, IEEE 802. 15. 4 protocol defines the physical layer (Physical Layer, PHY) and MAC layer ((Medium Access layer, MAC) communication standard, wherein the physical layer specifications define the main band wireless communication is used, and a data communication symbol rate of the band, MAC layer defines a plurality of wireless signals shared access and other air channel.

IEEE 802. 15.4 (ZigBee) to work in labor SM band defines two bands, 2. 4GHz band and 896 / $915 \mathrm{MHz}$ frequency bands. In the work IEEE 802. 15. 4 in total provides 27 channels: in 2. $4 \mathrm{GHz}$ band, a total of 16 channels, channel communication speed is $250 \mathrm{kbps}$; in the $915 \mathrm{MHz}$ band, a total of 10 channels, channel communication speed is $40 \mathrm{kbps}$; at $896 \mathrm{MHz}$ band, there is a channel, the channel communication rate of 20kbps. ZigBee network topology major star network and grid networks. Different network topologies correspond to different application areas in ZigBee wireless networks, different network topologies to configure network nodes have different requirements, the type of network node could be a coordinator, routers and end nodes, specific configuration need to profile decision ${ }^{[1]}$.

In the ZigBee network, according to the type of device can be ZigBee network equipment is divided into three types: coordinator, router and terminal node, the coordinator is responsible for the establishment of wireless networks, network topology information maintenance and network management functions; the router is responsible for network routing, network management functions requested; terminal node is generally not responsible for maintaining network information, only need to add a specific wireless network. The coordinator is a network set up by, at the beginning of the establishment of the network, first scan the radio frequency channel to see if there are the same types of networks exist, if not present, according to the characteristics of the network number and network channels to create a ZigBee wireless network; coordinator may be responsible for the network and to bind user-level communication mechanism. 


\section{Gateway Requirements Analysis}

Role perception layer of data acquisition and wireless transmission, generally use RFID, sensors, cameras for domestic, agricultural, transportation, water conservancy environmental data collection and transmission technology to transfer data via wireless transport layer using an existing Wi-Fi, 2G / 3G, broadband and other communication technologies, to expand the data transmission from the perception layer, network-aware and realize the combination of the communication network. the main application layer responsible for data processing, to provide users with a variety of services, is the ultimate goal of networking applications lies. Internet of Things gateway is located in the middle of the transport layer, connecting the sensor network and traditional communication network, its feature includes protocol conversion, data transceiver and network sensor management ${ }^{[2]}$. Fig.1 shows the gateway requirements analysis.

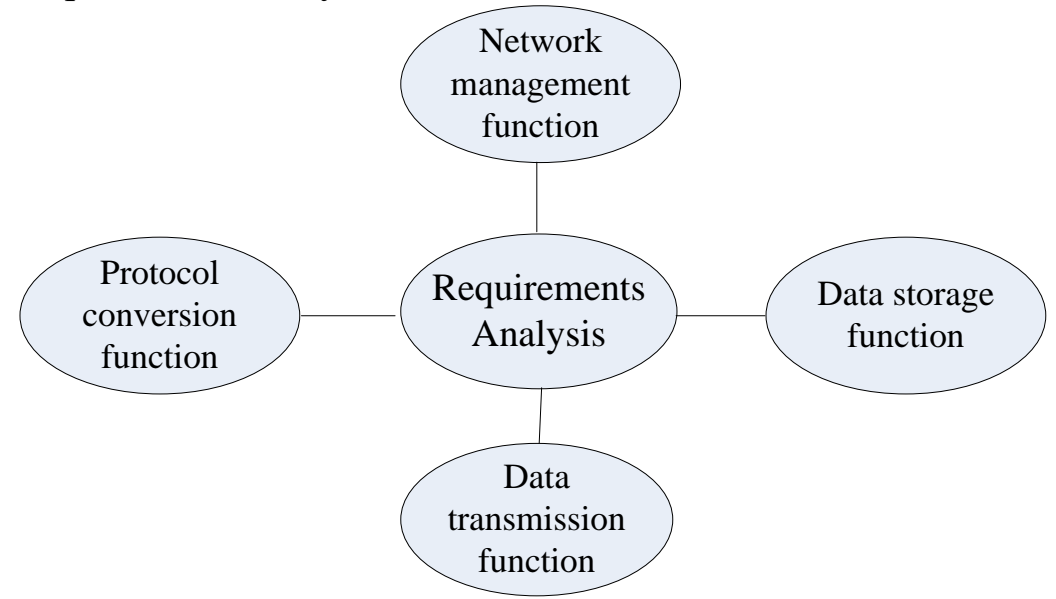

Fig. 1.The gateway requirements analysis

Gateway most basic function is to achieve the conversion between different protocols. Upon receiving ZigBee node sent data gateway based on ZigBee protocol specification removed PHY layer, MAC layer, the network layer header and application layer data act accordingly, and then follow the TCP / IP protocol standard re-encapsulating the data, and then sent via Ethernet; the opposite direction of data transmission also requires a similar process. Thus, the gateway should also have ZigBee and TCP / IP stack two protocols. Sensor network gateway as a communication bridge and communication networks, data transfer can play a role .ZigBee uploading data through the network after the Gateway can reach the host IP network, data the same IP network or control commands can also gateway to reach the appropriate ZigBee nodes.

ZigBee gateway should implement ZigBee network management, such as the establishment of the network, network application processing node, and assign a network address to maintain the network topology, the recovery of the node address to leave, etc. The gateway subsystem need to have data storage capabilities includes: according to the administrator to set a certain format to store real-time data collection, monitoring center for port after the transfer of view; setting information storage systems, each monitoring point parameter setting information management system, user information and other data. In order to meet the local monitoring center in the LAN environment of each monitoring point monitoring and management needs, the gateway subsystem needs to use an embedded Web server to ensure that their local area after the access, equivalent to the monitoring center to provide remote monitoring services. Development of the Internet has greatly changed people's way of life, the ZigBee network access TCP / IP network makes it through the Internet to monitor the environment, the access node for remote management and control, is currently a hot research area Internet of Things problems ${ }^{[3]}$. 


\section{Overall Hardware Design of Gateway}

Overall use dual CPU architecture including the CC2530 chip ZigBee module and control module 1 Godson chip design in two modules were used ZigBee Development Kits and Boards Godson 1B, take the suite as a node ZigBee network coordinator after, a coordinator with the Godson 1B development board connected together to complete the gateway .TI's CC2530 single-chip integrated SOC solutions, the RF transceiver, industrial strength 51 microcontroller core, Zstack protocol stack integrated on the same chip. Zstack protocol stack and open source stack as compared Freakz, more integrity and stability. the company is now free and open Zstack TI for developers to download and use, you can save a lot of costs.

MIPS-based streamlining Godson-1 chip instruction set, clocked at 200-233 MHz, it can be configured to $266 \mathrm{MHz}$, to meet the needs of the gateway processing performance compared with the mainstream ARM chips, with a higher price .1B development board peripherals rich, convenient debugging and development. DART uses serial port to connect the CC2530, and the Godson 1B two parts. things nodes collected more than some temperature, voltage and other sensor data, the transmission rate is not high, with serial fully able to meet the requirements. Fig.2 shows gateway platform core board physical.

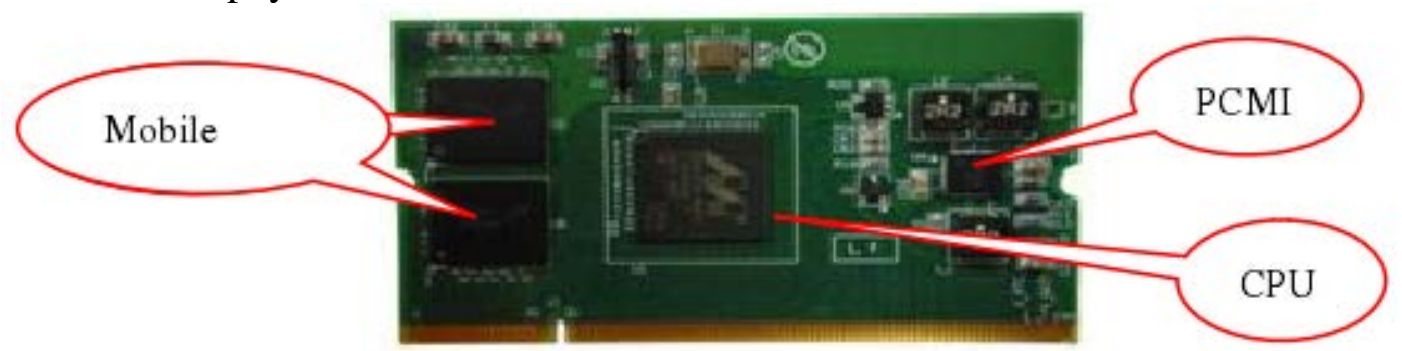

Fig. 2.The gateway platform core board physical

Gateway is Internet of Things hub for wireless sensor networks and the Internet, the Internet of Things should have a protocol conversion gateway function. And perception layer interaction, networking gateway uses ZIGB EE wireless protocol. Application layer interacts with Internet of Things gateway uses the TCP protocol. Internet of Things gateway according to their main function can be divided into three modules: 1 . ARM core module. ZIGBEE module is responsible for data collection to read, parse, store. 2. ZIGBEE module is responsible for receiving data from the sensor. 3. Network module is responsible for receiving a request for the application layer gateway Internet of Things, and the request for a corresponding response ${ }^{[4]}$.

\section{Gateway Software Design and Testing}

Gateway platform implant embedded Linux operating system at the core of the ARM microprocessor, all the programs running on the system, the system unified management. Run the program mainly consists of user space applications and kernel space drivers of two parts, the main function of the system to achieve the application, to communicate with other application processes, data exchange. Kernel space includes the various drivers and hardware communication bus for data communication, while providing the application software to operate the hardware module interfaces. Fig.3 shows the software design overall structure. 


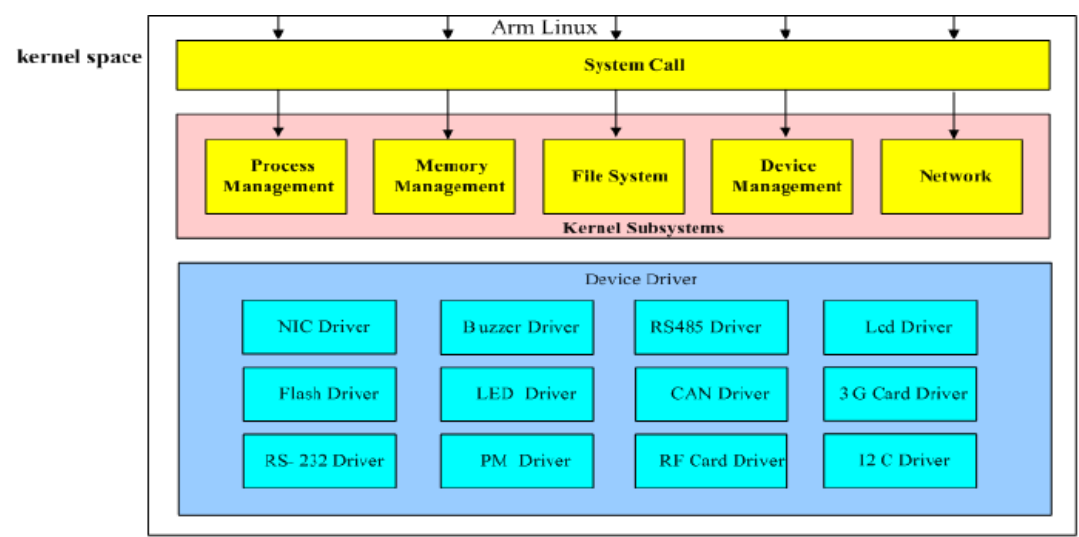

Fig. 3.The software design overall structure

The gateway system, as a network gateway platform is a communication client, remote or local monitoring centers as a server-side, both ends of the socket-based communication to achieve, since the IP address of the 3G network application is dynamic, and monitoring after the server uses a static IP address, so the server creates a socket and wait for the client's active connection in order to establish a communication link for data exchange ${ }^{[5]}$.

ZIGBEE wireless transmission having instability, but in the use of things in the gateway, this instability is permitted. Focuses on Ethernet module TCP packet loss module, insert the module test piles in the TCP program, the host PC statistical software packages corresponding request packet and response packet $\mathrm{P}$ are the same. If not the same as logged by the long run of things gateway and gateway running log statistics, the final conclusion. Gateway data distortion is acceptable data parsing software error. Things gateway for packet loss rate testing, get things thousandth gateway packet loss rate, negligible. So packet loss rate of Things gateway standard can be used stably. Data networking gateways distortion performance testing can obtain data networking gateways and no distortion. Description gateway software part of things, and no error analysis data portion, the sensor can accurately report the required data is parsed into PC data formats.

\section{Conclusions}

An integration between the ZigBee network and the IP network to the future development of Internet of Things, this paper presents a ZigBee gateway designs based on the basic functions of the gateway were realized experiments to verify the validity of the data collected .ZigBee node function through upload gateway to IP network PC client, the implementation of the ZigBee network management, protocol conversion, data transmission and other functions, to achieve two heterogeneous network interconnection purposes. Internet of Things gateway is a Internet of Things applications extremely important part, play a bridging role of the network/Internet and telecommunications networks between perception. This article describes a Zigbee-based embedded Internet of Things gateway system, enabling sensor networks and telecommunications networks, the Internet data transfer, protocol conversion and management control function, can be widely used in smart home, industrial monitoring, smart grid, environmental monitoring and other Internet of Things applications.

\section{References}

[1] Kim, Seong Hoon. ZigBee internet working architecture monitoring a multi-hot ZigBee network technology [J]. Consumer Electronics, 2009, 55(3): 1286-1294.

[2] Ahmad Akl, Chen Feng, Shahrokh Valaee. A Novel Accelerometer-Based Gesture Recognition System [J]. Signal Processing, 2011, 59(12): 6197-6205.

[3] Xiaoyan Xiong .Design of Wireless Sensor Networks Node Applied to Acquisition and Transmission of Vibration Signals [C]. Computational Aspects of Social Networks, Taiyuan, 2010, 693-696. 
[4] Wang Xudong. Filtering the acceleration signal in different algorithm for comparison and analysis [C]. Computational Problem-Solving (ICCP), Chengdu, 2011, 171-173.

[5] Malhi K, Mukhopadhyay, Subhas Chandra. A Zigbee-Based Wearable Physiological Parameters Monitoring System [J]. Sensors Journal, IEEE. 2012, 12(3):423-430. 